\title{
Letalidad de hongos entomopatogenos sobre Dysdercus peruvianus (Hemiptera: Pirrhocoridae)
}

\author{
Lethality of entomopathogenic fungi on Dysdercus peruvianus (Hemiptera: \\ Pyrrhocoridae)
}

Francesca Falconi, Aldo Flores y Pedro Castellanos

\begin{abstract}
Facultad de Ciencias Biológicas, Universidad Nacional Mayor de San Marcos, Av. Venezuela cdra 35 s/n Ciudad Universitaria. Apartado 110058, Lima 11, Perú.

Email Pedro Castellanos:

pcastellanoss@unmsm.edu.pe
\end{abstract}

Presentado: 22/11/2009

Aceptado: $\quad 09 / 04 / 2010$

Publicado online: $14 / 12 / 2010$

\begin{abstract}
Resumen
Se evaluó la patogenicidad y virulencia sobre Dysdercus peruvianus de una cepa de Acremonium y una de Scopulariopsis, aisladas de adultos infectados de D. peruvianus procedentes de Mala, Provincia de Cañete. También se evaluó una cepa de Beauveria sp. aislada de Schistocerca piceifrons peruviana procedente de Ayacucho. Los bioensayos se realizaron sobre ninfas del cuarto estadío de $D$. peruvianus y las concentraciones empleadas fueron $3,7 \times 10^{8}, 1,9 \times 10^{8}, 9,4 \times 10^{7}$ conidias/mL para Beauveria sp., Acremonium sp. y Scopulariopsis sp. respectivamente. Veinte días posteriores al tratamiento, los mayores porcentajes de mortalidad los causaron Beauveria sp. (83,3\%) y Acremonium sp. (80\%). Scopulariopsis sp. causo una mortalidad de $23,3 \%$. Acremonium sp. fue la cepa más agresiva con un tiempo de letalidad (TL50) 3,8 días.
\end{abstract}

Palabras clave: entomopatógeno, Dysdercus, Beauveria, Acremonium, Scopulariopsis, arrebiatado.

\section{Abstract}

We assessed the pathogenicity and virulence on Dysdercus peruvianus with a strain of Acremonium and one of Scopulariopsis isolated from infected adult $D$. peruvianus from Mala, Cañete, south of Lima. A strain of Beauveria sp., isolated from Schistocerca piceifrons peruviana from Ayacucho, was also evaluated. Bioassays were conducted on the fourth instar nymphs of $D$. peruvianus, and the concentrations used were $3,7 \times 10^{8}, 1,9$ $\mathrm{x} 10^{8}, 9,4 \times 10^{7}$ conidia / $\mathrm{mL}$ for Beauveria sp., Acremonium sp. and Scopulariopsis sp. respectively. At 20 days after treatment, the highest mortality rates were caused by Beauveria sp. (83,3\%) and Acremonium sp. (80\%). Scopulariopsis sp. caused a mortality of $23,3 \%$. Acremonium sp. was the more aggressive strain with a lethal time (LT50) of 3,8 days.

Keywords: entomopathogenic, Dysdercus, Beauveria, Acremonium, Scopulariopsis, cotton stainer.

\section{Introducción}

Los hongos entomopatógenos causan una alta mortalidad sobre sus hospederos (Lecuona et al. 2001, Thara et al. 2001, Liu et al. 2002). Algunos como los del género Metarhizium (Ascomycota), invaden directamente a su huésped a través de la cutícula por lo que son ampliamente utilizados en el de control biológico (Lubeck et al. 2008). Se han estimado cerca de 700 especies de hongos entomopatógenos en aproximadamente 90 géneros. Beauveria, Metarhizium, Lecanicillium e Isaria son los géneros fáciles de producir en masa y comercializados para control biológico. Sin embargo el grado de patogenicidad está en función de condiciones ambientales (Vega et al. 2009). El cultivo del algodón sufre el ataque de muchas plagas, siendo Dysdercus peruvianus Guérin-Méneville, 1831, el “arrebiatado”, una de las más comunes (Beingolea, 1973).

El objetivo del presente trabajo fue evaluar tres cepas de hongos: Beauveria sp. cepa PR-11 y dos hongos aislados de insectos adultos de Dysdercus peruvianus.

\section{Material y métodos}

Crianza de los insectos.- Adultos de Dysdercus peruvianus Guérin-Méneville, 1831 fueron recolectados de los cultivos de algodón de la localidad de Mala, Provincia de Cañete, al sur de Lima. Los insectos fueron mantenidos en envases con agua y alimentados con semillas de algodón y hojas de mandarina. Las semillas y el agua fueron renovadas cada 3 días. Los huevos de $D$. peruvianus fueron colocados sobre semillas de algodón y recolectados cuidadosamente.

Aislamiento e identificación de hongos.- Insectos adultos recolectados en campo fueron mantenidos en cautiverio en condiciones de alta densidad para estimular la expresión de las infecciones latentes que pudieran traer del campo; muertos los insectos, fueron sumergidos en una solución de hipoclorito al 5\% por 1 minuto y luego se lavaron tres veces en agua destilada para ser colocados en placas petri con algodón húmedo. A partir de estos ejemplares se aislaron cepas de hongos que se conservaron en Agar Papa Dextrosa (APD) hasta su evaluación entomocida. Los hongos fueron identificados en base a sus características morfológicas según Egorova (1980) y Barnet y Hunter (1998). Además se empleó la cepa de Beauveria sp. PR-11 aislada de Schistocerca piceifrons peruviana procedente de Ayacucho (Pariona et al. 2007).

Multiplicación de los hongos.- La suspensión de conidias usadas como inoculo fueron obtenidas de los cultivos de 21 días de incubación, crecidas sobre frascos con APD en pico de flauta a temperatura de laboratorio. Las conidias fueron cosechadas de los frascos por raspado agregando una solución estéril de agua destilada con Tween 80 (0,1\%). La concentración del inoculo fue calculada empleando la cámara de Neubauer.

Caracterización fisiológica de las cepas.- La caracterización fisiológica se realizó mediante la evaluación de producción de conidias y porcentaje de germinación.

Para la producción de conidias (esporulación) se inoculó $10 \mu \mathrm{L}$ de la solución conidial con una concentración de $10^{7}$ conidias/mL en placas petri con APD diseminándose en toda la superficie del medio con la ayuda de una espátula de Drigalsky esterilizada. Luego fueron mantenidas a temperatura de laboratorio durante 21 días; se utilizaron cuatro repeticiones para cada cepa. Transcurrido el tiempo se agregó una solución de 10 $\mathrm{mL}$ de agua destilada con Tween 80 al $0,1 \%$ y se dispersaron los conidios. Los conteos de conidios se realizaron en una cámara de Neubauer. 
El porcentaje de germinación (viabilidad) se realizó en placas petri conteniendo APD se marcaron 6 puntos en la superficie externa inferior de la placa. Se tomaron $5 \mu \mathrm{L}$ de diluciones $10^{-4}$ de cada cepa de hongo preparada para la prueba de conteo de conidias (previa agitación) y se depositaron en los puntos marcados, a razón de 6 alícuotas por placa (cada alícuota es una repetición para cada cepa de hongo evaluado). Las muestras fueron mantenidas a $22^{\circ} \mathrm{C}$, después de las 24 horas se agregó azul de lactofenol para detener la germinación y dar contraste a las conidias. Cada sector de agar que contenía una alícuota fue cortado y colocado en un portaobjeto y cubierto con un cubreobjeto. El conteo se realizó en 5 campos para cada repetición.

Bioensayos en Dysdercus peruvianus. - Los bioensayos con ninfas del cuarto estadio de $D$. peruvianus fueron realizadas en condiciones de laboratorio. La prueba de patogenicidad fue realizada por inmersión de 10 insectos en una suspensión conidial $\left(10^{8}\right.$ conidia $\left./ \mathrm{mL}\right)$ por aproximadamente 5 segundos. Para todos los tratamientos se empleo el mismo método de aplicación con tres repeticiones de 10 insectos. Los testigos fueron sumergidos en una solución de agua destilada estéril con Tween 80 al 0,1\%.

El ensayo consistió de 4 tratamientos: (T1) PR-11, (T2) DperMa052B, (T3) DperMa053M, (T4) testigo.

Luego del tratamiento las ninfas fueron transferidas individualmente en un contenedor plástico $(20 \mathrm{~mL})$ usando un pincel fino. Cada envase fue tapado, y para proveer de ventilación a las ninfas se realizaron pequeños agujeros en las tapas de los envases. Las ninfas fueron alimentadas con hojas de mandarina y semillas de algodón. El alimento fue renovado cada 2 días. La mortalidad de las ninfas fue registrada diariamente durante 20 días. Las ninfas muertas fueron colocadas en cámaras húmedas para facilitar el crecimiento de micelio, comprobándose así que el individuo estaba infectado. Para el análisis estadístico se utilizó un diseño completamente al azar, los datos obtenidos de mortalidad fueron sometidos a un análisis de varianza (ANOVA), para lo cual el porcentaje de individuos muertos fue transformado al arcoseno. Posterior al análisis de ANOVA se aplicó el test de Duncan $(\mathrm{p}<0,05)$ para comparar los promedios.

Tiempo de letalidad al 50 y $\mathbf{8 0 \%}$ (LT50 y LT80). - En este ensayo se determinó el tiempo necesario para matar el $50 \%$ y el $80 \%$ de cada población ninfal experimental por medio de una tabla de mortalidad acumulada diaria durante 20 días. Se realizó un análisis de varianza (ANOVA), comparándose los promedios mediante la prueba de Tukey (diferencia de significación) $(\mathrm{p}<0,05)$.

Tasa de infección.- Se seleccionaron las ninfas muertas a lo largo del experimento y se colocaron en cámara húmeda para realizar un conteo de ninfas infectadas por el hongo y así hallar el porcentaje dentro de cada unidad experimental y tratamiento. Se realizó un análisis de varianza (ANOVA) a los datos obtenidos comparándose los promedios mediante la prueba de Tukey (diferencia de significación) $(\mathrm{p}<0,05)$. La tasa de infección determinó el porcentaje de ninfas con confirmación de esporulación (micosis) al final del experimento.

\section{Resultados y discusión}

Aislamiento e identificación de cepas.- De las 50 cepas de hongos aisladas, dos cepas fueron seleccionadas para evaluar su patogenicidad. Estas cepas fueron: Acremonium sp. DperMa052B y Scopulariopsis sp. DperMa-053M. La infección fúngica natural por posibles hongos entomopatógenos en los insectos recolectados fue baja 9,8\%. Esta baja frecuencia de infección encontrada también fue reportada por Shah et al. (1997) quienes señalan que en la República de Benin (África) entre 1990 y 1992, se mantuvieron bajo cría masiva más de 23000 insectos, y se obtuvieron 79 aislamientos siendo 8 de ellos de $B$. bassiana (0,03\%) y 67 de Metharhizium flavoride (0,29\%).

Nuestros resultados permiten remarcar la presencia natural de hongos entomopatógenos y la factibilidad de obtener cepas fúngicas, a partir de insectos aparentemente sanos. Es importante destacar que los géneros Acremonium y Scopulariosis no fueron reportados anteriormente en el Perú como entomopatógenos y se desconoce sobre su distribución.

Conocidos hongos entomopatógenos como Beauveria spp. y Paecilomyces fumosoroseus no fueron aislados en nuestro trabajo, a diferencia de Samson et al. (1984) que si encontraron B. bassiana frecuentemente en insectos troglobios. Otro entomopatógeno como Metarhizium anisopliae tampoco fue encontrado. Esta especie solo tiene ocurrencia sobre coleópteros (Domsch et al. 1993).

\section{Caracterización morfológica de los hongos}

Beauveria sp. cepa PR-11.- La cepa de Beauveria sp. presentó una colonia con micelios blancos de aspecto algodonoso y superficie semielevada. Se observó la formación de sinemas reportadas por Vargas (2003) para Beauveria bassiana y por Pariona (2007) para Beauveria sp. En el cultivo produjo un pigmento de color amarillo, que evidenció la producción de la toxina Bauvericina (Gómez 1998, Vargas 2003). También fueron observadas células conidióforas formando racimos, irregularmente densos. El conidióforo se forma desde una base hinchada adelgazándose hacia la porción que sostiene la célula conidiófora, la cual se presenta en forma de zig - zag, las conidias se forman en cada punto de flexión. Las conidias son hialinas, ovoides, globosas, unicelulares y pequeñas. Estas características coinciden con las descripciones de Gómez (1999) y Bustillos (2001).

Acremonium sp. cepa DperMa-052B.- Presentó una colonia con micelios blancos de aspecto algodonoso y superficie elevada. Produjo pigmentos de color liliáceo que se difundió en el medio. Las hifas eran septadas, delicadas y hialinas, con conidióforos largos y delgados, que dan origen a microconidias hialinas, unicelulares y elipsoidales, reunidas en una cabezuela en el extremo distal del conidióforo; características del género Acremonium.

Scopulariopsis sp. cepa DperMa-053M.- Este hongo presentó una colonia con micelio marrón, superficie plana y crecimiento moderado. Produjo un pigmento de color mostaza que se difundió en el medio. Presentó condióforos generalmente ramificados o produciendo en el ápice racimos de conidias, dejando anelaciones en el extremo. Las conidias, llamadas en este caso anelosporas, fueron hialinas, unicelulares, globosas con la base truncada, producidas en cadenas basipetales.

\section{Caracterización fisiológica del hongo}

Recuento de conidias.- Las tres cepas evaluadas presentaron una alta producción de conidias después de 21 días de sembrado. Las diferencias en la producción de conidias de los tres hongos evaluados fueron altamente significativas (ANOVA, $\mathrm{p}<0,05$ ), con valores mayores a $10^{7}$ conidias $/ \mathrm{mL}$. La cepa de Beauveria sp. PR-11 presentó el valor más alto con $4,6 \times 10^{8}$ conidias/ 
mL, seguido de la cepa de Acremonium sp. DperMa-052B con $2,9 \times 10^{8}$ conidias $/ \mathrm{mL}$. El valor más bajo lo presentó la cepa $S c o-$

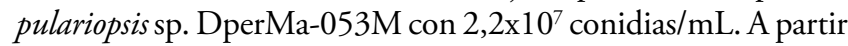
de estos resultados podríamos considerar a Beauveria sp. PR-11 como la cepa más promisoria ya que en iguales condiciones de temperatura, humedad y tiempo de incubación produjo mayor cantidad de conidias.

Los hongos evaluados presentaron valores altos de esporulación con un porcentaje de 92,24\% para Beauveria sp. PR-11, 95,12\% para Acremonium sp. DperMa-052B y 94,90\% para Scopulariopsis sp. DperMa-053M. No se observaron diferencias significativas entre los porcentajes de esporulación (ANOVA, $\mathrm{p}<0,05$ ). Los porcentajes de germinación obtenidos a las 24 horas coinciden con los valores considerados para el control de calidad de hongos entomopatógenos ( $85 \%$ en $24 \mathrm{~h}$ ) señalado por Gómez (1998), para que al asperjar el hongo en el campo, tenga un rápido efecto sobre la plaga y un corto período de exposición a condiciones ambientales (Alean 2003).

Prueba de patogenicidad.- De los tres hongos evaluados, dos produjeron porcentajes de mortalidad superiores al 50\% sobre las ninfas del cuarto estadio de D. peruvianus, con mortalidades

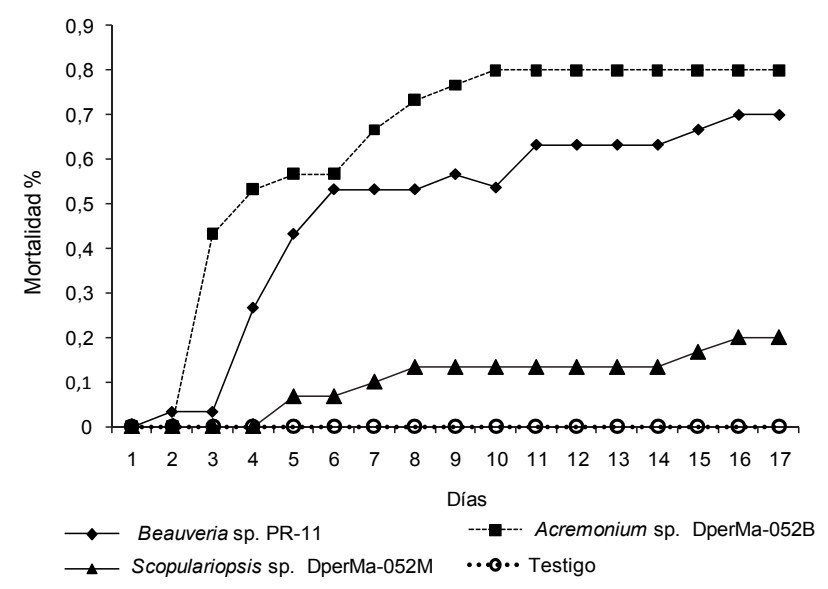

Figura 1. Mortalidad diaria causada por las cepas de los hongos evaluados sobre ninfas del $4^{\text {to }}$ estadio de Dysdercus peruvianus.

promedio al finalizar el estudio de $83,3 \%$ para Beauveria sp. PR11 y $80 \%$ para Acremonium sp. DperMa-052B. Scopulariopsis sp. DperMa-053M presentó una mortalidad baja sobre el insecto que alcanzo solo el 23,3\% de los insectos tratados (Fig. 1).

Al decimo día no se observaron diferencias significativas en las mortalidades producidas por las tres cepas evaluadas. Al decimoquinto día las cepa Beauveria sp. PR-11 produjo el 56,67\% de mortalidad y Acremonium sp. DperMa-052B el 80\%; siendo significativamente diferentes a Scopulariopsis sp. DperMa-053M que produjo sólo $16,67 \%$ de mortalidad, existiendo diferencias significativas. Al finalizar el estudio los tres hongos presentaron valores significativamente diferentes al testigo (Duncan, $P<0,05$; Tabla 1). No fue necesario aplicar la formula de Abbot para corregir los datos de mortalidad acumulada debido a que no se registro mortalidad en los testigos.

Teniendo en cuenta los resultados de porcentaje de mortalidad, la cepa Beauveria sp. PR-11 fue la más virulenta sobre ninfas de $D$. peruvianus ya que obtuvo un mayor valor. Este resultado muestra una patogenicidad inespecífica del hongo, por no tener una relación con el hospedero de origen. Respecto a este hecho, Feng et al. (1994) señalan que en general cepas de B. bassiana, tienden a mostrar gran virulencia sobre su hospedante original o sobre especies cercanamente relacionadas, tal como lo reportado por Romaña y Fargues (1987), quienes observaron que solo las cepas aisladas a partir de Hemiptera fueron altamente patógenas a Rhodnius prolixus. Sin embargo Moorhouse et al. (1993) sugieren que la especificidad hospedero patógeno no es tan estricto; esto explicaría los resultados de alta virulencia presentada por la cepa heteróloga Beauveria sp. PR-11 aislada del ortóptero Schistocerca peruviana sobre las ninfas de D. peruvianus, así como fue el caso de una cepa aislada del lepidóptero Diatraea saccharalis que causó $97 \%$ de mortalidad sobre el hemíptero Triatoma infestans (Klug) (Lecuona et al. 2001).

Los insectos muertos por el hongo Acremonium sp. DperMa052B presentaron una coloración oscura causada posiblemente por sustancias similares a la oosporeína, para el caso de infecciones con Beauveria (Lecuona 1990, Khachatourians 1996). Al realizar la cámara húmeda para determinar la tasa de infección por el hongo la recuperación no ocurrió sobre todos los cadáveres. Por esta razón, no hubo una relación proporcional entre los porcentajes de mortalidad y esporulación, lo que coincide con lo observado en otras especies de insectos (Rodríguez et al. 2006), resultando que la mortalidad por micosis (con confirmación de esporulación) es menor a la mortalidad real (coloración oscura).

Esta menor capacidad de esporulación de Acremonium sp. DperMa-052B podría indicar que la mortalidad fue causada por la actividad de alguna sustancia fúngica segregada durante la fase de ligero crecimiento vegetativo (Jaronski 1997, Inglis et al. 2001, Shah y Pell 2003) y que luego el hongo presenta dificultades para atravesar el tegumento y emerger a la superficie como pone de manifiesto el bajo porcentaje de insectos que mostraron micosis. Esta característica de una posible producción de toxinas, es interesante para su posterior investigación en el desarrollo de formulaciones de productos insecticidas. Es importante señalar que el hecho de no esporular no invalida a las cepas para ser empleadas como bioinsecticidas (Luna Rodríguez $\&$ Lecuona 2000). La baja mortalidad producida por el hongo

Tabla 1. Tasa de mortalidad total causadas por las cepas de Beauveria sp., Acremonium sp. y Scopulariopsis sp.

\begin{tabular}{|c|c|c|c|c|c|c|c|c|}
\hline \multirow{2}{*}{ Tratamiento } & \multicolumn{2}{|c|}{ Día 5} & \multicolumn{2}{|c|}{ Día 10} & \multicolumn{2}{|c|}{ Día 15} & \multicolumn{2}{|c|}{ Día 20} \\
\hline & $\%$ & arcsen & $\%$ & arcsen & $\%$ & arcsen & $\%$ & arcsen \\
\hline PR-11 & 43,33 & $0,72 \mathrm{~B}$ & 56,67 & $0,85 \mathrm{~B}$ & 66,67 & $0,95 \mathrm{~B}$ & 83,33 & $1,15 \mathrm{C}$ \\
\hline DperMa-052B & 56,67 & $0,85 \mathrm{~B}$ & 80,00 & $1,11 \mathrm{~B}$ & 80,00 & $1,11 \mathrm{~B}$ & 80,00 & $1,11 \mathrm{C}$ \\
\hline DperMa-053M & 6,67 & $0,26 \mathrm{~A}$ & 13,33 & $0,37 \mathrm{AB}$ & 16,67 & $0,42 \mathrm{~A}$ & 23,33 & $0,50 \mathrm{~B}$ \\
\hline Testigo & 0,00 & $0,00 \mathrm{~A}$ & 0,00 & $0,00 \mathrm{~A}$ & 0,00 & 0,00A & 0,00 & $0,00 \mathrm{~A}$ \\
\hline
\end{tabular}

Valores con la misma letra no presentan diferencias significativas según el análisis de Duncan $(P<0,05)$. ANOVA $(P<0,05)$. 
Tabla 2. Tiempo letal al 50 y 80\% (TL50 y TL80) de la población de las cepas evaluadas

\begin{tabular}{lcc} 
Tratamiento & TL 50 & TL 80 \\
PR-11 & $5,6^{\mathrm{a}}$ & 19,2 \\
DperMa-052B & $3,8 \mathrm{~B}$ & 10 \\
\hline
\end{tabular}

Valores con la misma letra no presentan diferencias significativas según el análisis de Tukey $(P<0,05)$. ANOVA $(P<0,05)$

Scopulariopsis sp. sobre el arrebiatado plantea la necesidad de utilizar concentraciones de conidias más elevadas de este hongo a fin de obtener una mayor virulencia. El ensayo realizado en el presente estudio fue en condiciones de temperatura y humedad de laboratorio, sin embargo los resultados obtenidos fueron satisfactorios; donde las cepas Beauveria sp. PR-11 y Acremonium sp. DperMa-052B eliminaron al doceavo día post tratamiento entre 63 y $80 \%$ de la población tratada, respectivamente con concentraciones de $10^{8}$ conidias $/ \mathrm{mL}$.

Letalidad al 50 y $80 \%$ de la población $\left(\mathrm{TL}_{50}\right.$ y $\left.\mathrm{TL}_{80}\right)$.Se realizó el análisis de varianza (ANOVA) a los hongos que alcanzaron mortalidad mayor al $50 \%$ que fueron Beauveria sp. PR-11 y Acremonium sp. DperMa-052B. En el análisis de varianza (ANOVA) el tiempo necesario para eliminar al 50\% de la población es altamente significativo $(\mathrm{p}<0,01)$. Siendo el TL50 de 5,6 días para Beauveria sp. PR-11 y de 3,8 días para Acremonium sp. DperMa-052B. Según el análisis de Tukey la diferencia entre las dos cepas es significativamente diferente, donde la cepa Acremonium sp. DperMa-052B, mostró ser más efectiva actuando en menor tiempo (3,8 días) en promedio (Tabla 2 ).

El tiempo necesario para eliminar el $80 \%$ de la población fue de 19,2 días para Beauveria sp. PR-11 y de 10 días para Acremonium sp. No se pudo realizar un análisis de varianza (ANOVA) para el $\mathrm{TL}_{80}$ debido a la falta de datos para el hongo Acremonium sp. DperMa-052B en el que solo en una de las tres repeticiones superó el $80 \%$ (Tabla 2 ).

Se observaron diferencias respecto a la agresividad de las cepas evaluadas, ya que cuando se determinó el tiempo de letalidad para el 50\% (TL50) de la población tratada, Acremonium sp. necesitó sólo 3,8 días, mientras que Beauveria sp. necesitó de 5,6 días. Así mismo, el pico de mortalidad alcanzada por la cepa de Acremonium sp. (80\%) fue sólo al décimo día post tratamiento, mientras que Beauveria sp. obtuvo la mayor mortalidad (83,3\%) recién al vigésimo día post tratamiento. Esto demuestra que la cepa evaluada de Acremonium sp. es más agresiva que la de Beauveria sp.; sin embargo, la cepa que presento mayor virulencia fue el hongo Beauveria sp. si consideramos el porcentaje de mortalidad alcanzada sobre $D$. peruvianus.

Tasa de infección.- La aparición de micelio ocurrió a partir de las 48 horas de muerte del insecto al ser colocado en cámara húmeda. Cuando el hongo esporuló en los cadáveres de las ninfas del arrebiatado, adquirieron un aspecto pulverulento. Según el análisis de varianza (ANOVA), la variable ninfas infectadas presentó valores altamente significativos $(\mathrm{p}<0,01)$ para Beauveria sp. PR-11, mientras que para los hongos Acremonium sp. DperMa052B y Scopulariopsis sp. DperMa-053M el valor de esta variable fue significativo $(\mathrm{p}<0,05)$. Para la variable ninfas infectadas el tratamiento Beauveria sp. PR-11 fue la que causo el mayor porcentaje de ninfas infectadas $(96,3 \%)$, siendo significativamente diferente a los tratamientos Acremonium sp. DperMa-052B y Scopulariopsis sp. DperMa-053M, en los que los porcentajes de infección fueron de 37,14\% y 33,3\% respectivamente (Tabla 3).

La cepa de Beauveria sp. mostró una mayor capacidad de esporulación en comparación a los otros dos hongos evaluados sobre los cadáveres de D. peuvianus, este comportamiento observado tiene una importancia practica para la aplicación del hongo como bioinsecticida ya que si las condiciones son apropiadas, podría incrementar la densidad del propágulo infectivo en el campo, trasmitiendo el inóculo horizontalmente y prolongar el tiempo de infección (Ayasse \& Paxton 2002).

\section{Literatura citada}

Alean I. 2003. Evaluación de la patogenicidad de diferentes hongos entomopatogenos para el control de la mosca blanca de la yuca Aleurotrachelus socialis Bondar (Homoptera: Aleyrodidae) bajo condiciones de invernadero. Trabajo de grado presentado como requisito parcial para optar al título de Microbióloga Agrícola y Veterinaria. Pontificia Universidad Javeriana. Facultad de Ciencias Básicas Microbiología Agrícola y Veterinaria. Colombia.

Ayasse M. \& R. Paxton. 2002. Brood protection in social insects. In: Hilker M, Meiners T (eds). Chemoecology of insect eggs and egg deposition. Blackwell, Berlin, Germany. pp. 117-148.

Barnett H.L \& B. Hunter. 1998. Ilustrate Genera of Imperfec Fungi. 4ta Edición. The American Phytopathological Society. St Paul, Minnesota (USA). 217pp.

Bustillos A. 2001. Hongos en insectos y posibilidades de uso en el control biológico de plagas en Colombia. En: Seminario sobre uso de entomopatógenos en Colombia. Sociedad Colombiana de Entomología. Bogotá. p: 30-53.

Domsch K.H., W. Gams, T. Anderson. 1993. Compendium of soil fungi. Vol. I [reprint]. - 860 p. Eching. En A. Kubatova, L. Dvorak, 2005. Entomopathogenic fungi associated with insects hibernating in underground shelters. Czech Mycol. 57(3-4): 221-237.

Egorova L.N. 1980. Hongos del suelo del Este. Hyphomycetes. Instituto de Biología del Suelo. Centro Científico del Lejano Este. Academia de Ciencias. URSS. p: 49-76.

Feng M.G., T.J. Poprawsky \& G.C. Khachatourians. 1994. Production, formulation and application of the entomopathogenic fungus Beauveria bassiana for insect control: Current Status. Biocontrol Science and Technology. 4: 3-34.

Tabla 3. Tasa de infección de los hongos evaluados

\begin{tabular}{cccccc}
\hline Cepa & Tasa de infección $\%$ & ANOVA $(\mathbf{P}<0,05)$ & Sig. & DE & Rango \\
\hline PR-11 & 96,297 & s & A & 6,414 & $88,89-100,00$ \\
DperMa-052B & 37,143 & S & B & 7,561 & $28,57-42,86$ \\
DperMa-053M & 33,333 & s & B & 34,171 & $0,00-50,00$ \\
\hline
\end{tabular}

Valores con la misma letra no presentan diferencias significativas según el análisis de Tukey $(P<0,05)$. ANOVA $(P<0,05)$. Sig. = Significancia. $s=$ significativo 
Gómez H. 1998. Importancia de los hongos entomopatógenos. En Nuevos Aportes del Control Biológico en la Agricultura sostenible. Editores: A. Lizarraga T., U. Barreto C. y J. Hollands. Red de Acción e Alternativas al uso de Agroquímicos. p: 97-112.

Gómez H. 1999. Aislamiento e identificación de hongos entomopatógenos de la mosca blanca Bemisia tabaci (Gennadius) (Homoptera: Aleyrodiae) en Lima, Perú. Revista peruana de entomología 41: 83-86.

Inglis G.D., M.S. Goettel, T.M. Butt, H. Strasser. 2001. Use of Hyphomycetous fungi for managing insect pests. Fungi as Biocontrol Agents: progress, problems and potential (Butt, T.M., Jackson, C. y N. Magan Eds.). CABI Publishing. pp. 23-69.

Jaronski S.T. 1997. new paradigms in formulations mycoinsecticides. Pesticide Formulations and Appications Systems. 17va Volume. ASTM STP. 1328. pp. 93-112.

Kachatourians G.G. 1996. Biochemestry and Molecular biology of entomopathogenic fungi. En Luna Rodriguez, J. A. \& Lecuona, R. E. 2002. Selección de cepas de hongos entomopatógenos nativos para el control de la tucura Rhammatocerus pictus (Bruner) (Orthoptera: Acrididae). RIA, INTA, Argentina. 31 (1):67 - 84.

Lecuona R. 1990. El control microbiano como regulador de insectos plaga. Instituto Nacional de Tecnología Agropecuaria INTA. Agricultura Sostenible. Publicación No 4.

Lecuona R., J. Edelstein, M. Berretta, F. La Rosa \& A. Arcas. 2001. Evaluation of B. bassiana (Hyphomycetes) Strains as Potencial Agents for Control of Triatoma infestans (Hemiptera:Reduviidae). Journal of Medical Entomology. 38(2): 172-179.

Liu H., M. Skinner, L. Parker, M. Brownbridge. 2002. Pathogenicity of Beauveria bassiana, Metarhizium anisopliae (Deuteromycotina: Hyphomycetes), and other Entomopathogenic Fungi Against Lygus lineolaris (Hemiptera: Miridae) Journal of Economic Entomology. 95(4): 675-681.

Luna Rodriguez J.A. \& R.E. Lecuona. 2002. Selección de cepas de hongos entomopatógenos nativos para el control de la tucura Rhammatocerus pictus (Bruner) (Orthoptera: Acrididae). RIA, INTA, Argentina. 31 (1):67 - 84.

Lubeck I., W. Arruda, B. K. Souza, et al. 2008. Evaluation of Metarhizium anisopliae strains as potential biocontrol agents of the tick Rhipicephalus (Boophilus) microplus and the cotton stainer Dysdercus peruvianus. Fungal Ecology 1(2): 78-88.
Moorhouse S.R., A.T. Gillespie \& A.K. Charnley. 1993. Laboratory selection of Metarhizium spp. Isolates for control of vine weevil larvae (Otiorhynchus sulcatus). Journal Invertebrate Pathology. 62: $15-21$.

Pariona N., P. Castellanos, E. Leon. 2007. Capacidad entomocida de cepas nativas de Beauveria sp. sobre schistocera piceifrons peruviana (Lynch Arribalzaga, 1903). Revista Peruana de Biología. 14(2): 253-257.

Rodríguez M., M.E. Gerding \& A. France. 2006. Selección de aislamientos de hongos entomopatógenos para el control de la polilla del tomate (Tuta absoluta Meyrick) (Lepidoptera: Gelechiidae). Agricultura Técnica. (Chile). 66:159-165.

Romaña C., \& J. Fargues. 1987. Sensibilité des larves del hémiptére hématophage, Rhodnius prolixus (Triatominae) aux hyphomycéte entomopathogénes. Entomophaga. 32: 167 -179 .

Samson R.A., M.C. Rombach, K.A. Seifert. 1984. Hirsutella guignardii and Stilbella kerviellei, two troglobiotic entomogenous Hyphomycetes. Persoonia 12: 123-134.

Shah P.A., C. Kooyman \& A. Paraiso. 1997. Surveys for fungal pathogens of locust and grasshoppers in Africa and Near East. En Goettel, M. and Johnson D. (Eds). Microbial Control of Grasshoppers and Locusts. Mem. Entom. Soc. Can. 171: 27-35.

Shah P.A., J.K. Pell. 2003. Entomopatogenic fungi as biological control agents. Applied Microbiology and Biotechnology. 61: 413-423.

Thara F., K. Yaginuma, N. Kobayashi, K. Mishiro, T. Sato. 2001. Screening of entomopathogenic fungi against the brownwinged green bug, Plautia stali Scott (Hemiptera: Pentatomidae) Appl. Entomol. Zool. 36(4): 495-500.

Vargas M. 2003. Caracterización de tres cepas de Beauveria brongniartii (Saccardo), Petch y su virulencia sobre Phthorimaea operculella (Zeller) y Symmetrischema tangolias (Gyen). Tesis para obtener el Título Profesional de Biólogo, mención en Microbiología y Parasitología. Universidad Nacional Mayor de San Marcos. Lima, Perú.

Vega F.E., S.M. Goettel, M. Blackwell, et al. 2009. Fungal entomopathogens: new insights on their ecology. Fungal Ecology 2(4): 149-159. 


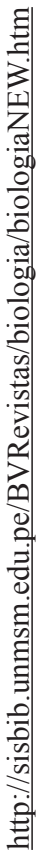

